\title{
Spectrophotometric Determination of Trace Phosphate Ions by Amplitude-Modulated Flow Analysis Coupled with Malachite Green Method
}

\author{
Takeshi Uemura, ${ }^{*}$ Takeshi Ogusu, ${ }^{* *}$ Masaki TaKeuchI, ${ }^{* * *}$ and Hideji TanaKa $* * * \dagger$ \\ *Graduate School of Pharmaceutical Sciences, Tokushima University, 1-78-1 Shomachi, \\ Tokushima 770-8505, Japan \\ **Faculty of Pharmaceutical Sciences, Tokushima University, 1-78-1 Shomachi, Tokushima 770-8505, Japan \\ ***Institute of Health Biosciences, Tokushima University, 1-78-1 Shomachi, Tokushima 770-8505, Japan
}

\begin{abstract}
An amplitude-modulated flow analysis coupled with lock-in detection is proposed for the determination of trace phosphate. The flow rate $F_{\mathrm{S}}$ of sample solution is varied in response to a periodic signal $V_{\mathrm{c}}$ of alternating waveform. A coloring reagent solution (ammonium molybdate + Malachite Green) is delivered at a constant flow rate $F_{\mathrm{R}}$. Under the constant total flow rate $F_{\mathrm{T}}$, both solutions are merged with a diluent (water). Phosphate ion in the sample reacts with molybdate and then with Malachite Green to form green ion pairs in acidic media. Downstream, the absorbance of the mixed solution is measured at $625 \mathrm{~nm}$. The output voltage $V_{\mathrm{d}}$ from the detector is sent to a lock-in amplifier, where the wave component of $V_{\mathrm{d}}$ that has the same frequency as that of $V_{\mathrm{c}}$ is distinguished from background signals. Phosphate ion can be determined from the amplitude of the component thus extracted. The calibration curve is linear $\left(r^{2}>0.998\right)$ and the

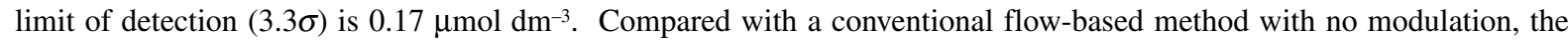
present method is less susceptible to the baseline drift due mainly to the adsorption of the ion-pair on the optical window, because the quantification is based not on the absorbance itself but on the amplitude of the absorbance. Good recoveries around $100 \%$ are obtained for the phosphate ions spiked into real water samples.
\end{abstract}

(Received April 16, 2010; Accepted May 6, 2010; Published July 10, 2010)

\section{Introduction}

Phosphorus is an essential element for living organisms, and a ubiquitous component of environmental samples that relates especially to the ecosystem. Phosphorus compounds have been extensively applied to fertilizers, pesticides and functional materials (sensor, catalyst, artificial bone). The element, together with nitrogen, is known to cause eutrophication in aquatic environments. On the other hand, phosphorus resources will be depleted in the near future. ${ }^{1}$ It is, therefore, important to determine trace levels of phosphate ions in order to grasp its behavior and cycle precisely. During water analyses, phosphorus is often classified into orthophosphate, acid-hydrolyzable phosphate, and total phosphorus; ${ }^{2}$ more detailed classifications are described in the literature. ${ }^{3}$ A lot of studies have been carried out for the determination of various kinds of phosphorus species, as reviewed by Worsfold et al., ${ }^{3}$ Estela and Cerdà, ${ }^{4}$ Morais et al. ${ }^{5}$ and Spangler et al. ${ }^{6}$ Orthophosphate ion (hereafter, phosphate ion or $\mathrm{Pi}$ ) is generally determined by Molybdenum Blue spectrophotometry without pretreatments such as acid hydrolysis and acid digestion. The lower determinable concentration of the method is around $\mu \mathrm{mol} \mathrm{\textrm {dm } ^ { - 3 }}$ to sub- $\mu \mathrm{g} \mathrm{dm}^{-3}$ level. Various methods including spectrophotometry and chemiluminescence method have been proposed for the

† To whom correspondence should be addressed.

E-mail: htanaka@ph.tokushima-u.ac.jp determination of trace phosphate ion. The limit of detection has been lowered down to nmol dm${ }^{-3}-$ sub-nmol dm${ }^{-3}$ level through the combination with preconcentration techniques. ${ }^{7,8}$

In the present paper, we propose an amplitude-modulated flow analysis (AMFA) coupled with lock-in detection for the determination of trace phosphate ions in water samples. The AMFA is a simplified variation of the amplitude-modulated multiplexed flow analysis (AMMFA), recently developed by our group. ${ }^{9}$ The main purpose of the modulation in the former is to change the signal of interest to a periodic wave that can be separated from background signals for trace analysis, whereas the purpose in the latter is the multiplexing of information so that the multiplexed signal can be deconvoluted into each contribution for simultaneous analysis. The flow rate of sample solution is varied in response to a periodic voltage signal $V_{\mathrm{c}}$. While the total flow rate is held constant, the sample is merged with reagent and diluent solutions. Downstream, the analytical signal for the mixed solution, $V_{\mathrm{d}}$, is monitored. The signal contains information on the analyte as the amplitude of the wave component that has the same frequency as that of $V_{\mathrm{c}}{ }^{9}$ This piece of information can be filtered out from background noises through lock-in amplification. Malachite Green method $^{10,11}$ is selected as the color reaction for phosphate ion, because the method can give about 10 times higher analytical signal than the Molybdenum Blue method. The proposed AMFA makes it possible to perform sensitive determination of phosphate ion $\left(\operatorname{LOD}(3.3 \sigma)=0.17 \mu \mathrm{mol} \mathrm{dm}^{-3}\right)$ with reasonable precision $\quad\left(\mathrm{RSD}=1.1 \% \quad\left(n=6, \quad C_{\mathrm{Pi}}=2.9 \mu \mathrm{mol} \mathrm{dm}-3\right)\right)$. 

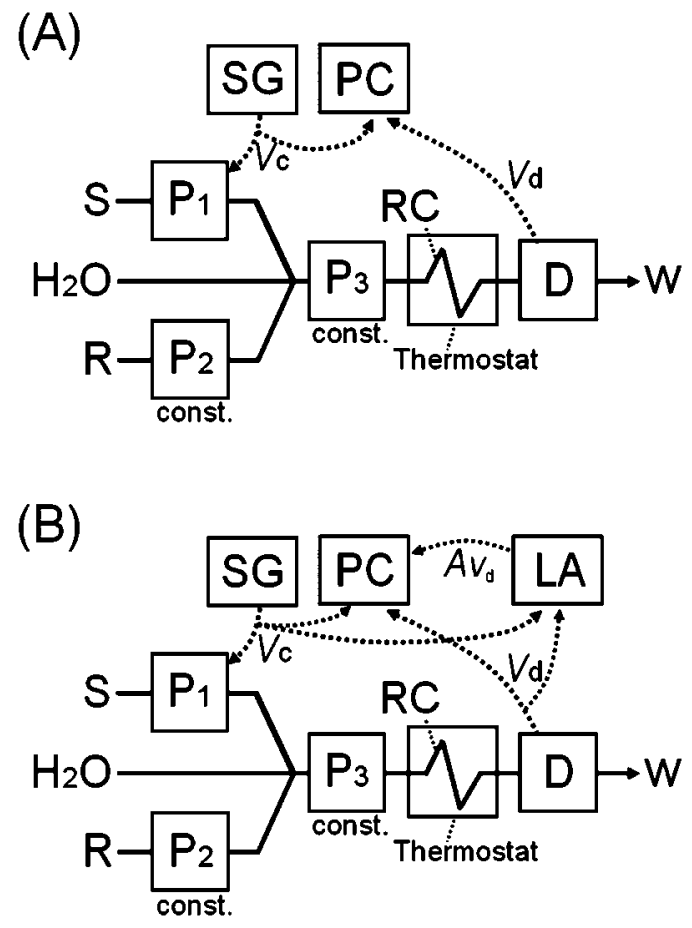

Fig. 1 Schematic diagram of flow systems. (A) Amplitudemodulated flow analysis (AMFA) with fast Fourier transform. (B) AMFA with lock-in detection. S, Sample solution; R, reagent solution; $\mathrm{H}_{2} \mathrm{O}$, water (diluent); w, waste; $\mathrm{P}_{1}, \mathrm{P}_{2}$ and $\mathrm{P}_{3}$, peristaltic pumps; RC, reaction coil; $\mathrm{D}$, spectrophotometer; $\mathrm{SG}$, signal generator; $\mathrm{PC}$, laptop computer with a card type A/D-D/A converter; LA, lock-in amplifier; $V_{\mathrm{c}}$, controller output voltage; $V_{\mathrm{d}}$, detector output voltage; $A_{\mathrm{Vd}}$, amplitude of $V_{\mathrm{d}}$ component having the same frequency as that of $V_{\mathrm{c}}$.

The method is applicable to the analyses of environmental water samples.

\section{Experimental}

\section{Flow system}

Figure 1 shows the schematic diagram of the flow systems developed in the present study: AMFA systems with (A) fast Fourier transform (FFT) and with (B) lock-in detection. A lock-in amplifier (LA; NF Co. 5640, Japan) was employed for the latter system. Three peristaltic pumps $\left(\mathrm{P}_{1}, \mathrm{P}_{2}\right.$ and $\mathrm{P}_{3}$; Rainin Dynamax RP-1, USA) were used for delivering solutions. Pharmed tubings with $0.51 \mathrm{~mm}$ i.d. were used for $\mathrm{P}_{1}$ and $\mathrm{P}_{2}$ as pump tubes and tubing with $0.79 \mathrm{~mm}$ i.d. was used for $\mathrm{P}_{3}$. The flow rate of sample solution $S$ delivered using $P_{1}$ was varied in response to the controller output voltage $V_{\mathrm{c}}$ generated from a signal generator (SG; NF Corp. WF1974, Japan). The $V_{\mathrm{c}}$ was of an alternating current waveform that ranged from 0 to $2 \mathrm{~V}$, corresponding to the sample flow rate from 0.0 to $0.5 \mathrm{~cm}^{3} \mathrm{~min}^{-1}$, with a period of typically $15 \mathrm{~s}$. The flow rate of coloring reagent solution delivered using $\mathrm{P}_{2}$ was kept constant at $0.6 \mathrm{~cm}^{3} \mathrm{~min}^{-1}$. Both solutions were merged with a diluent (water) at the confluence point (polypropylene joint), while the total flow rate was held constant at $1.2 \mathrm{~cm}^{3} \mathrm{~min}^{-1}$ by $\mathrm{P}_{3}$. The reason for this constancy was to keep constant the lag time between the mixing of the three solutions $\left(\mathrm{S}, \mathrm{R}\right.$ and $\left.\mathrm{H}_{2} \mathrm{O}\right)$ and the sensing of the mixed solution by a detector (D; Soma Optics, S3250 visible spectrophotometer, Japan). This lag time consisted mainly of the physical transit time of the mixed solution from the confluence point to the detector, and to a lesser degree included the response time of the detector. The solution was then introduced to a reaction coil RC $(1 \mathrm{~mm}$ i.d. and $1 \mathrm{~m}$ long for Manifold A; $0.5 \mathrm{~mm}$ i.d. and $0.5 \mathrm{~m}$ long for Manifold B) in order to accelerate color reaction by the heating. The temperature of $\mathrm{RC}$ was controlled with a temperature sensor (TOP L-TN-4-PT100, Japan), a silicon rubber heater (TOP $5099-01,100 \times 25 \mathrm{~mm}$ ) and a temperature controller (Toho BX-303, Japan). The reacted solution was introduced to a specially made quartz flow cell (Soma Optics; optical path length $5 \mathrm{~mm}$ ) set in the detector. The cell was designed in order that the air bubbles accidentally introduced to the cell could pass through it without being trapped. The absorbance of the mixed solution was measured with the detector at 700 and $625 \mathrm{~nm}$ for Molybdenum Blue and Malachite Green methods, respectively. The output signal from the detector $(1.0 \mathrm{~V}$ per absorbance unit) was quantized by an A/D-D/A converter (Measurement Computing PC-CARD-DAS16/12-AO, USA). Resulting digital data were acquired in a laptop computer (PC; Toshiba DynaBook Satellite 1850 SA120C/4, Japan) at the sampling frequency of $10 \mathrm{~Hz}$ and stored as the Microsoft Excel format. A program written in Visual BASIC in-house was used to acquire data, analyze them and graphically display the results automatically.

\section{Color reaction}

Two kinds of color reactions (i.e., Molybdenum Blue and Malachite Green methods) were examined in the present study. The reagent for the former was $1.2 \times 10^{-3} \mathrm{~mol} \mathrm{dm}^{-3}$ ammonium molybdate solution containing $2.0 \times 10^{-2} \mathrm{~mol} \mathrm{dm}^{-3}$ ascorbic acid, $\quad 1.8 \times 10^{-4} \mathrm{~mol} \mathrm{dm}^{-3}$ potassium antimonyl tartrate, $1.1 \times 10^{-2} \mathrm{~mol} \mathrm{dm}^{-3}$ ammonium amidosulfate and $0.4 \mathrm{~mol} \mathrm{dm}^{-3}$ sulfuric acid. The reagent for the latter was $1.6 \times 10^{-2} \mathrm{~mol} \mathrm{dm}^{-3}$ ammonium molybdate solution containing $4.0 \times 10^{-3} \mathrm{~mol} \mathrm{dm}^{-3}$ Malachite Green, $0.5 \%$ polyvinyl alcohol (polymerization degrees; 2000) and $1.3 \mathrm{~mol} \mathrm{dm}^{-3}$ sulfuric acid. The polyvinyl alcohol was added as a stabilizer for the ion-pairs formed from phosphomolybdate and Malachite Green. ${ }^{10}$

\section{Reagents}

All the reagents used in the present study were of analytical reagent grade, purchased from Kanto Chemicals (Tokyo, Japan), Nacalai Tesque (Kyoto, Japan) or Wako Pure Chemical Industries (Osaka, Japan). The reagents were used without further purification. Zartorius Arium 611DI deionized water was used throughout. Real samples were collected from Yoshino river (fresh and tidal areas) and Akui river in Tokushima Prefecture, Japan. The samples were each filtered through a disposable disk filter with a pore size of $0.45 \mu \mathrm{m}$, purchased from Kanto Chemical Co. (Tokyo, Japan), and then each was stored in a polypropylene bottle in a refrigerator.

\section{Results and Discussion}

\section{AMFA-FFT}

Initially, the Molybdenum Blue method, ${ }^{2}$ the most common method for phosphate determination, was examined as a color reaction. Manifold A shown in Fig. 1 was used for this spectrophotometry. Frequency analysis was carried out by FFT. $^{9}$ The coloration of Molybdenum Blue is slow, typically taking $10 \mathrm{~min}$ or more. This slow reaction inevitably requires a long reaction coil, resulting in the decrease in the amplitude of modulated signals due to the axial dispersion of the sample in the flow system. The influence of reaction temperature was, 
therefore, studied in the range from 33 to $86^{\circ} \mathrm{C}$ in order to accelerate the coloration. In this experiment, the aqueous solution of $8.4 \times 10^{-2} \mathrm{mmol} \mathrm{dm}^{-3} \mathrm{KH}_{2} \mathrm{PO}_{4}$ was delivered as a sample solution at the constant flow rate of $0.5 \mathrm{~cm}^{3} \mathrm{~min}^{-1}$. The absorbance of the solution increased with the temperature, as expected. However, when the temperature is high, the formation of air bubbles in the conduit became frequent. The noises that were caused by the bubbles resulted in errors in FFT analysis. We selected, therefore, $50^{\circ} \mathrm{C}$ as the optimal reaction temperature because enough absorbance, comparable to that available by the official method (batch mode), was obtained at this temperature.

A calibration curve was obtained in the range from 0.000 to $0.084 \mathrm{mmol} \mathrm{dm}^{-3}$ by measuring various concentrations of phosphate standard solutions. The flow rate of the solutions was each varied with sinusoidal control voltage $(0-2 \mathrm{~V})$ having the period of $30 \mathrm{~s}$. This period was set as the length of FFT window. Eight data points at the same intervals in $30 \mathrm{~s}$ were used for FFT computation, as described in detail elsewhere. The amplitude of the fundamental wave component $\left(A_{\mathrm{Vd}}\right)$ of $V_{\mathrm{d}}$ corresponds, therefore, to the concentration of phosphate ions in the solutions. The linear regression for the calibration curve was obtained as follows:

$$
A_{\mathrm{Vd}}=3.040 C_{\mathrm{Pi}}-0.026
$$

where $C_{\mathrm{Pi}}$ is the concentration of phosphate ions. The linearity of the calibration curve was good $\left(r^{2}=0.998\right)$ and the limit of detection LOD $(3.3 \sigma)$ was $0.021 \mathrm{mmol} \mathrm{dm}^{-3}$. The obtained LOD was, however, not so sufficient compared with that by the official method. The dispersion of colored sample solution in the flow system is considered to be the main reason for the damping of the amplitude, and hence for the decrease in the sensitivity. Even if FFT computation was carried out after the measurement using as many as $2048\left(=2^{11}\right)$ data points within $120 \mathrm{~s}$ (4 periods of $V_{\mathrm{c}}$ and thus $V_{\mathrm{d}}$ ), the amplitude for $0.021 \mathrm{mmol} \mathrm{dm}^{-3}$ phosphate ion was not so distinct.

\section{AMFA-lock-in detection}

In order to determine sub- $\mu \mathrm{mol} \mathrm{dm}{ }^{-3}$ level of phosphate ion, we applied a Malachite Green method ${ }^{10,11}$ as a color reaction. Manifold B shown in Fig. 1 was used for this purpose. The ion-pair formation between molybdophosphate and Malachite Green is rapid, which makes it possible to shorten the reaction coil length. A narrower $(0.5 \mathrm{~mm}$ i.d.) and shorter $(0.5 \mathrm{~m}$ long $)$ reaction coil was, therefore, used in order to reduce the damping of the amplitude. The residence time of the mixed solution in the reaction coil in Manifold B was as low as $4.9 \mathrm{~s}$, in contrast with $39.3 \mathrm{~s}$ in Manifold A. Furthermore, the lock-in amplifier, a specialized instrument for picking up signal components of desired frequencies, was introduced for the frequency analysis we expected better sensitivity of amplitude than that available by FFT. The amplifier extracts the amplitude of the wave component that has the same frequency as that of $V_{\mathrm{c}}$. The effect of reaction temperature on the amplitude $A_{\mathrm{Vd}}$ for $15.5 \mu \mathrm{mol} \mathrm{dm}{ }^{-3}$ phosphate ion was investigated in the range from 25 to $60^{\circ} \mathrm{C}$. The $V_{\mathrm{c}}$ period was set at $30 \mathrm{~s}$. The $A_{\mathrm{Vd}}$ thus obtained was found to increase with the temperature and then slightly decreased after reaching the maximum at $50^{\circ} \mathrm{C}$. Therefore, $50^{\circ} \mathrm{C}$ was selected as the optimum reaction temperature.

The effect of $V_{\mathrm{c}}$ period $(5,10,15,20$ and $30 \mathrm{~s})$ on the sensitivity (i.e. amplitude) was investigated. The aqueous solution of $2.9 \mu \mathrm{mol} \mathrm{dm}{ }^{-3} \mathrm{KH}_{2} \mathrm{PO}_{4}$ was used as a sample solution. The $A_{\mathrm{Vd}}$ was found to increase with the period and reached a plateau at the period higher than $15 \mathrm{~s}$. In the range of the period shorter than $15 \mathrm{~s}$, the change of analyte concentration

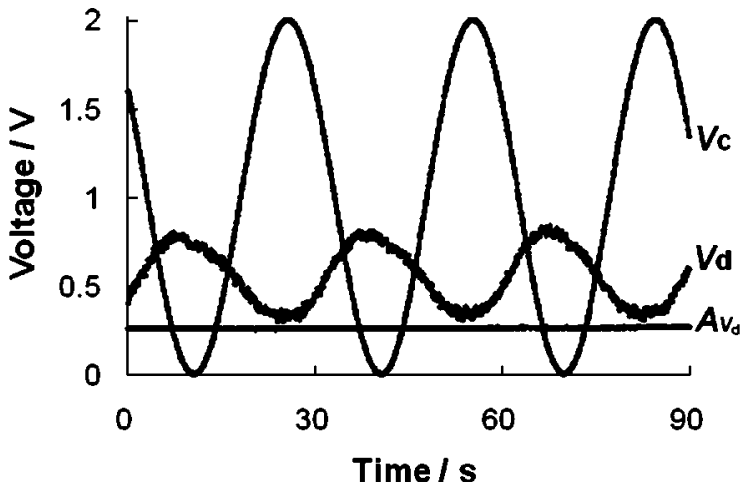

Fig. 2 Typical flow signals of AMFA-lock-in detection. $V_{\mathrm{c}}$, Controller output voltage; $V_{\mathrm{d}}$, detector output voltage; $A_{\mathrm{Vd}}$, amplitude of $V_{\mathrm{d}}$ component having the same frequency as that of $V_{\mathrm{c}}$. Concentration of phosphate ions in the sample: $2.9 \mu \mathrm{mol} \mathrm{dm}^{-3}$.

along the axial direction of the conduit is too steep to be followed by the detector. Moreover, in such condition, only subtle dispersion (diffusion and mixing) in the liquid phase tends to level the gradient in concentration, resulting in the damping of amplitude. These factors are considered to contribute to the lower amplitude in the range of the period shorter than $15 \mathrm{~s}$. A shorter period was, however, preferable with respect to sample throughput. That is, the sample to be introduced to the system should be invariable at least one period of $V_{\mathrm{c}}$ for frequency analysis. Therefore, $15 \mathrm{~s}$ was selected as the optimum period of the $V_{\mathrm{c}}$.

Figure 2 shows typical flow signals for the determination of $2.9 \mu \mathrm{mol} \mathrm{dm}^{-3}$ phosphate ion. The control signal $V_{\mathrm{c}}$ used for controlling the flow rate of sample solution is of a sinusoidal profile with a period of $15 \mathrm{~s}$ and an amplitude of $2 V_{\mathrm{p}-\mathrm{p}}(0-2 \mathrm{~V})$. Analytical signal $V_{\mathrm{d}}$ shows a similar shape to $V_{\mathrm{c}}$. The $V_{\mathrm{d}}$ did not reach to $0 \mathrm{~V}$ because of the damping of the amplitude mainly due to the axial dispersion of the sample solution. The difference in the phases between $V_{\mathrm{c}}$ and $V_{\mathrm{d}}$ is explained in terms of the lag time between the mixing of solution at the confluence point and its being sensed by the detector. The amplitude $A_{\mathrm{Vd}}$, corresponding to the concentration of phosphate ions in the sample, was stable at $0.26 \pm 0.01 \mathrm{~V}(n=900)$.

\section{Analytical performance of AMFA-lock-in detection}

Figure 3 shows calibration curves for phosphate ion in the

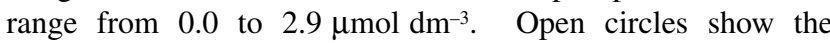
amplitude $A_{\mathrm{Vd}}$ (in voltage) obtained through the AMFA with lock-in detection at the optimum conditions described above. A conventional non-segmented continuous flow analysis was also applied as a reference, where the sample flow rate was not modulated but was held constant at $0.5 \mathrm{~cm}^{3} \mathrm{~min}^{-1}$. The results are shown by the open triangles in this figure. The linear regression lines (solid lines) obtained through the respective methods are $A_{\mathrm{Vd}}=40.41 C_{\mathrm{Pi}}+0.02\left(r^{2}=0.999\right)$ and $V_{\mathrm{d}}=8.47 C_{\mathrm{Pi}}+0.00\left(r^{2}=0.967\right)$. The slope of the former is higher than that of the latter, because $A_{\mathrm{Vd}}$ was outputted from the amplifier after 50 times amplification. The amplitudes before the amplification are plotted as open squares on the broken line in Fig. 3 for comparison. The respective LOD $(3.3 \sigma)$ values estimated from the calibration curves are 0.17 and $1.03 \mu \mathrm{mol} \mathrm{dm}^{-3}$. These values are much lower than that obtained by the AMFA-FFT method $\left(21 \mu \mathrm{mol} \mathrm{dm}{ }^{-3}\right)$. It is clear that the proposed method (AMFA-lock-in detection) is superior to the reference method (non-segmented continuous flow 


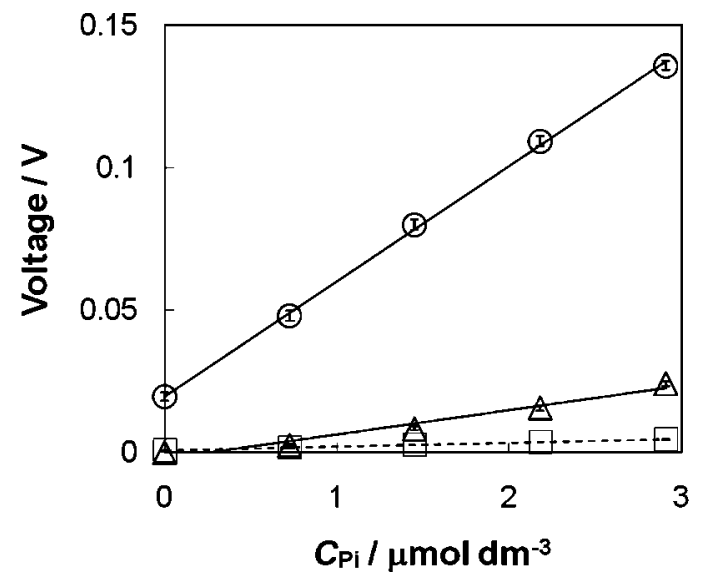

Fig. 3 Calibration curves for the determination of phosphate ions by Malachite Green method. Open circles, amplitude (in voltage) obtained from AMFA with lock-in detection (proposed method). Open triangles, conventional non-segmented continuous flow analysis with no modulation. In the present system, the amplitude was outputted after automatic amplification by the lock-in amplifier. Thus, the amplitudes before the amplification were calculated and also plotted in this figure as open squares in order to make a comparison between the present method and the conventional method.

analysis) with respect to linearity $\left(r^{2}\right)$ and sensitivity (LOD). In the latter method, the calibration curve slightly curved upwards. In addition, it showed hysteresis (data are not shown here). It was observed that the optical cell was liable to be tinged greenish. When the conduit was cleaned with 2-propanol between each sample measurement, the linearity and the sensitivity for the continuous flow analysis were improved to

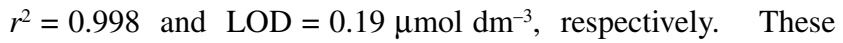
results suggested that the green ion-pair that formed from phosphomolybdate and Malachite Green was adsorbed on the inner surface of the optical flow cell, and caused baseline drift during the measurements. In contrast with such conventional methods, in which the determination is based on the absorbance value itself, the present method is less susceptible to the baseline drift because the determination is based on the amplitude of the absorbance. These facts are considered to contribute to higher linearity and lower LOD of the present AMFA with lock-in detection.

\section{Effect of baseline drift on AMFA-lock-in detection}

In order to ascertain the advantage of the amplitude modulation, we investigated the effect of baseline drift. Various concentrations $\left(0,1.3,2.6,3.8\right.$ and $\left.5.1 \mu \mathrm{mol} \mathrm{dm}{ }^{-3}\right)$ of Fast Green (FG), a food dye that has the maximum absorption wavelength of $625 \mathrm{~nm}$, were added to the sample solutions containing $2.9 \mu \mathrm{mol} \mathrm{dm}{ }^{-3}$ phosphate ion. The duration time for each sample solution was $c a .150 \mathrm{~s}$. The results are shown in Fig. 4, where $V_{\mathrm{d}}$ and $A_{\mathrm{Vd}}$ obtained through AMFA with lock-in detection are plotted as a function of time. The figure shows that the $V_{\mathrm{d}}$ drifted upwards with the concentration of FG. Nevertheless, $A_{\mathrm{Vd}}$ was almost constant $(0.23 \pm 0.01 \mathrm{~V}$ $(n=7500))$ regardless of the FG concentration. The results support the above-mentioned consideration that the proposed AMFA is less susceptible to the baseline drift than the conventional method with no modulation.

Effect of coexisting ions

The effect of diverse coexisting ions on the determination of

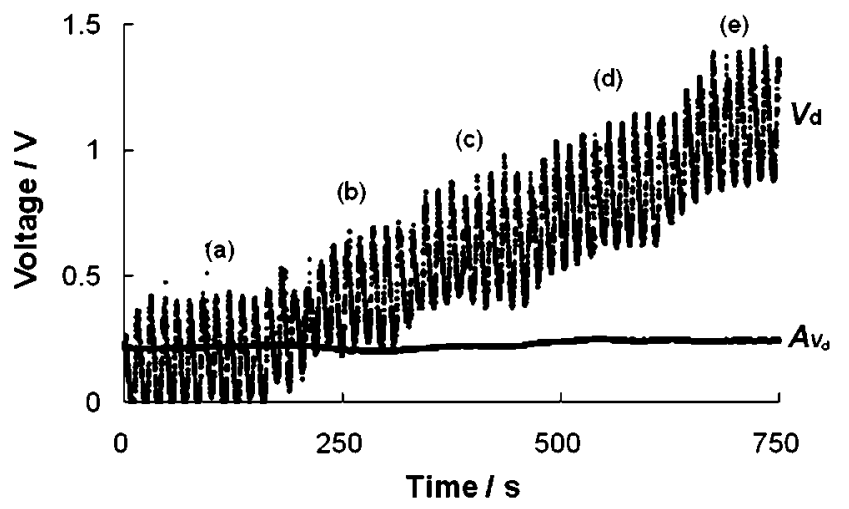

Fig. 4 Effect of baseline drift on analytical signal and amplitude. $V_{\mathrm{d}}$, Detector output voltage; $A_{\mathrm{Vd}}$, amplitude of $V_{\mathrm{d}}$ component having the same frequency as that of $V_{c}$. Concentration of Fast Green: $a, 0 ; b, 1.3$;

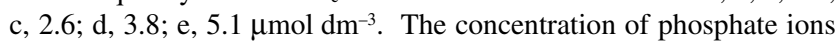

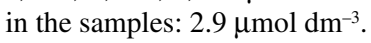

Table 1 Influence of coexisting ions

\begin{tabular}{|c|c|c|c|}
\hline Cation $^{\mathrm{a}}$ & $\begin{array}{c}\text { Tolerable limit/ } \\
\text { mol dm }{ }^{-3}\end{array}$ & Anion $^{\mathrm{b}}$ & $\begin{array}{c}\text { Tolerable limit } \\
\text { mol dm}^{-3}\end{array}$ \\
\hline $\mathrm{Na}^{+}$ & 0.1 & $\mathrm{Cl}^{-}$ & 0.1 \\
\hline $\mathrm{K}^{+}$ & 0.001 & $\mathrm{HCO}_{3}^{-}$ & 0.01 \\
\hline $\mathrm{NH}_{4}{ }^{+}$ & 0.001 & $\mathrm{NO}_{2}^{-}$ & 0.01 \\
\hline $\mathrm{Mg}^{2+}$ & 0.01 & $\mathrm{NO}_{3}^{-}$ & 0.001 \\
\hline $\mathrm{Ca}^{2+}$ & 0.003 & $\mathrm{SO}_{4}{ }^{2-}$ & 0.01 \\
\hline $\mathrm{Fe}^{3+}$ & 0.0001 & & \\
\hline
\end{tabular}

a. Added as chloride.

b. Added as sodium salt.

Tolerable limit: $5 \%$ error level.

phosphate ion was investigated. Various concentrations of cations (as chlorides) and anions (as sodium salts) were each added to $2.9 \mu \mathrm{mol} \mathrm{dm}{ }^{-3}$ phosphate solution. The tolerance limit of the coexisting ion was defined as the highest concentration that yielded an error below $5.0 \%$ in the analytical signal. The results are listed in Table 1 . The tolerable limits of the ions tested were almost in the same magnitudes as those reported in the literature. ${ }^{11,12}$ The concentration of the ions in environmental surface waters are generally lower than the tolerable limits listed in Table 1. The present method is, therefore, expected to be applicable to the determination of phosphate ions in environmental water samples.

\section{Application to real water samples}

The proposed method was applied to the determination of phosphate ions in real water samples. Recovery tests were carried out by spiking a known concentration of phosphate ion $\left(0,1\right.$ or $\left.2 \mu \mathrm{mol} \mathrm{dm}{ }^{-3}\right)$ to the sample solution. The non-spiked and spiked sample solutions were measured by the proposed method. The results obtained are listed in Table 2 . The concentrations of phosphate ion originally present in the samples are ranging from 0.17 to $0.33 \mu \mathrm{mol} \mathrm{dm}{ }^{-3}$. These values are equal to or higher than $\operatorname{LOD}(3.3 \sigma)(0.17 \mu \mathrm{mol} \mathrm{dm}-3)$ and lower

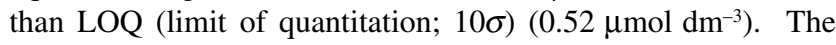
official method in batch mode (Molybdenum Blue method) ${ }^{2}$ was also applied to the non-spiked samples in order to acquire the reference values. No phosphate ion could, however, be detected, because the concentration of phosphate ions was too low to be 
Table 2 Determination of phosphate ions in river water samples

\begin{tabular}{cccc}
\hline Sample & $\begin{array}{c}\text { Added/ } \\
\mu \mathrm{mol} \mathrm{dm}\end{array}$ & $\begin{array}{c}\text { Found/ } \\
\mu \mathrm{mol} \mathrm{dm}^{-3}\end{array}$ & Recovery, \% \\
\hline Yoshino river (fresh area) & 0 & $\left(0.1_{7}\right)$ & - \\
& 1 & $1.1_{6}$ & 96.6 \\
Yoshino river (tidal area) & 0 & $2.2_{3}$ & 101.6 \\
& 1 & $\left.1.3_{3}\right)$ & - \\
Akui river (fresh area) & 2 & $2.3_{0}$ & $103_{3}$ \\
& 0 & $\left(0.3_{0}\right)$ & - \\
& 1 & $1.3_{0}$ & $100_{.0}$ \\
\hline
\end{tabular}

Sampling date: November 25, 2009.

Phosphate ion was added as $\mathrm{NaH}_{2} \mathrm{PO}_{4}$.

Analytical values lay in the range from LOD to LOQ are shown in the parentheses.

detected by the official method (LOQ: $1.05 \mu \mathrm{mol} \mathrm{dm}{ }^{-3}$ ). ${ }^{2}$ Good recoveries of $100 \pm 5 \%$ were obtained for all spiked samples. It was concluded, therefore, that the proposed method is easily applicable to the determination of trace phosphate ion at sub- $\mu \mathrm{mol} \mathrm{dm}{ }^{-3}$ level in real water samples.

\section{Conclusions}

An amplitude-modulated flow analysis (AMFA) with lock-in detection has been proposed for the determination of trace phosphate ions in water samples. Through the combination with a sensitive Malachite Green method, LOD can be lowered to $0.17 \mu \mathrm{mol} \mathrm{dm}{ }^{-3}$. In contrast with conventional spectrophotometries based on absorbance value itself, the proposed method is less susceptible to the baseline drift because this approach is based on the amplitude of absorbance. The system is rather simple and suitable for automation. The consumption of sample and reagent solutions is low at $c a$. 0.13 and $0.15 \mathrm{~cm}^{3}$ per 1 period of $V_{\mathrm{c}}(15 \mathrm{~s}$ : minimum requirement for one measurement). Currently, we are studying on the improvement in sensitivity by the introduction of air-segmentations. The results will be published in the near future.

\section{Acknowledgements}

This research was partly supported by a Grant-in-Aid for Exploratory Research (19655027), a Grand-in-Aid for Scientific Research (A) (20241020) and a Grand-in-Aid for Scientific Research (C) (21550083) from the Japan Society for the Promotion of Science (JSPS). The authors are grateful to Prof. Shoji Motomizu and Prof. Mitsuko Oshima, Okayama University, for their useful suggestions on the Malachite Green method.

\section{References}

1. P. H. Abelson, Science, 1999, 283, 2015.

2. JIS K 0101, "Kougyo-yosui Shiken Hoho (Testing Methods for Industrial Water, in Japanese)", 1998; K 0102, "Kogyohaisui Shiken Hoho (Testing Methods for Industrial Wastewater, in Japanese)", 2008, Japanese Industrial Standards Committee, Tokyo, Japan.

3. P. J. Worsfold, L. J. Gimbert, U. Mankasingh, O. N. Omaka, G. Hanrahan, P. C. F. C. Gardolinski, P. M. Haygarth, B. L. Turner, M. J. Keith-Roach, and I. D. McKelvie, Talanta, 2005, 66, 273.

4. J. M. Estela and V. Cerdà, Talanta, 2005, 66, 307.

5. I. P. A. Morais, I. V. Tóth, and A. O. S. S. Rangel, Talanta, 2005, 66, 341 .

6. C. Spangler, M. Schaeferling, and O. S. Wolfbeis, Microchim. Acta, 2008, 161, 1.

7. S. Motomizu and Z-H Li, Talanta, 2005, 66, 332.

8. P. J. Worsfold, I. D. McKelvie, and G. Hanrahen, "Advances in Flow Injection Analysis and Related Techniques", ed. S. D. Kolev and I. D. McKelvie, 2008, Elsevier, Amsterdam, $706-710$.

9. H. Tanaka, T. Mima, M. Takeuchi, and H. Iida, Talanta, 2008, 77, 576 .

10. S. Motomizu, T. Wakimoto, and K. Toei, Analyst, 1983, 108,361 .

11. S. Motomizu, T. Wakimoto, and K. Toei, Talanta, 1983, 30, 338.

12. A. Muñoz, F. Mas Torres, J. M. Estela, and V. Credo, Anal. Chim. Acta, 1997, 350, 21. 